\title{
Strength and Balance Training for Preventing Falls in Prostate Cancer Patients Receiving Androgen Deprivation Therapy: Case Report
}

Parivash Jamrasi ${ }^{1}$ MSc, Kyunghee Lee ${ }^{1}$ MSc, Kyoungmin Noh ${ }^{1}$ MSc, Juhyun Park ${ }^{2}$ MD, PhD, Hyeon Jeong ${ }^{3}$ MD, PhD, Sangjun Yoo $^{3} \mathrm{MD}$, PhD, Wook Song ${ }^{1,4} \mathrm{PhD}$

${ }^{1}$ Health and Exercise Science Laboratory, Institute of Sport Science, Seoul National University, Seoul; ${ }^{2}$ Department of Urology, Ulsan University College of Medicine, Asan Medical Center, Seoul; ${ }^{3}$ Department of Urology, Seoul National University College of Medicine, SMG-SNU Boramae Medical Center, Seoul; ${ }^{4}$ Institute on Aging, Seoul National University, Seoul, Korea

PURPOSE: Prostate cancer (PCa) survivors are at risk of falls due to the accelerated physiological changes following receiving androgen deprivation therapy (ADT). Thus, follow-up care of PCa survivors after treatment is vital to prevent high healthcare costs of fall-induced injuries and maintain their quality of life (QOL). Yet, we do not know what type of exercise can be beneficial to decrease falls risk factors in PCa patients. Here we report for the first time the possible advantage of strength and balance training (SBT) program on key components of falls predictors such as balance confidence, physical functioning performance and musculoskeletal health that might lead the next researcher to test this hypothesis on larger group of subjects.

METHODS: For this purpose, center-based SBT exercise with resistance band conducted twice a week for 8 weeks and we compared outcomes of bone health, physical functioning performance, balance confidence, fatigue and quality of life of two cases with PCa with one control subject at the baseline and after intervention period.

RESULTS: Following the exercise intervention, improvements were observed in bone mineral density (BMD), functional movement screen (FMS), backward walk and timed up and go (TUG) tests as well as outcomes of self-reported functional assessment of chronic illness therapy-fatigue (FACT-F) and functional assessment of cancer therapy-prostate (FACT-P); however, we could not declare the effects of SBT on lower body strength and power.

CONCLUSIONS: The 8-weeks intervention had positive impact on some falls risk factors (i.e., bone health, functional and balance performance) as well as QOL for participants of SBT program. However due to small number of participants we could not apply further quantitative analysis methods to make any conclusion on the efficacy of the intervention.

Key words: Geriatric Oncology, CancerTreatment, Falls, Exercise

\section{INTRODUCTION}

PCa and unintentional falls are considered as two serious public health problems for aging population [1]. A recent study determined that PCa survivors who received ADT had experienced falls more than unexposed men [2]. Although the exact mechanisms by which ADT increases risk of falls are not fully understood; suppression of androgen might be one of the possible explanations that describe this event. Decreased lower body muscle mass and strength, cognitive decline, worsen fatigue and progressive loss of BMD following initiating ADT could put PCa survivors at higher risk of impaired physical functioning performance and subsequently developing falls [3,4]. Subsequently a higher prevalence of fracture following ADT in PCa patients has been reported in studies [3, 5]. Thus, in order to prevent high costs of healthcare following fall-in-

Corresponding author: Wook Song Tel +82-10-6426-5726 Fax +82-2-880-7791 E-mail songw3dasnu.ac.kr

Keywords Geriatric Oncology, Cancer Treatment, Falls, Exercise, Prostate Cancer

Received 17 Jun 2020 Revised 13 Oct 2020 Accepted 21 Oct 2020

@) This is an Open Access article distributed under the terms of the Creative Commons Attribution Non-Commercial License (https://creativecommons.org/licenses/by-nc/4.0// which permits unrestricted non-commercial use, distribution, and reproduction in any medium, provided the original work is properly cited. 
duced injuries in older men with PCa, follow-up after treatment with purpose of enhancing physical function and maintaining body composition is vital to help survivors live longer.

Exercise is well-known as an economic and key adjuvant treatment in clinical oncology that improves QOL of cancer survivors. Many studies focused on investigating the benefits of exercise interventions on preventing falls and improving physical performance of older adults [6]. Recent physical activity guideline recommended that strength and balance are two necessary component of exercise program for older adults to prevent falls and disability [7]. Combination of strength and functional balance exercise has been demonstrated as an effective method to improve balance performance, rather than strength training alone $[8,9]$. Yet, no study demonstrated the effect of fall-prevention exercise tailored for PCa patients receiving ADT. To our knowledge the present study is the first clinical trial that verified the effects of 8-week SBT on falls risk factors such as balance confidence, physical functioning performance and muscle strength and musculoskeletal health in purpose of reducing the future health-care costs associated with falls.

\section{CASE DESCRIPTION}

This case study involved three subjects. All of them volunteered to participate in our project and according to their willingness, subject A \& $B$ participated in the supervised SBT exercise program, while subject C was clustered as an exercise placebo control subject. The baseline demographic characteristics of all cases are presented in Table 1.

Case A was a 74-year-old man, diagnosed with T3b N1 M0 non-small prostate cancer cell with a grade 4 gland according to Gleason grading system (Gleason score $4+4$ ) six years prior to our study. He underwent a radical retropubic prostatectomy in 2013 and began ADT with $10.8 \mathrm{mg}$ of Goserelin since April 2015; So, he had a history of 50-month ADT before starting the intervention. He also diagnosed with diabetes mellitus (DM). He was neither smoker nor regular alcohol drinker. He implied he does regular exercise by his own, and according to IPAQ his level of physical activity in one week before starting the intervention categorized as "moderate" or 1,173 MET-minutes per week. He was the only case who stated that had a fall experience while he was riding bicycle which resulted a surgery followed by limited range of motion on his left shoulder. He asserted that doctor recommendation was the only reason of participating on this study.

Case B a 77-year-old was diagnosed with grade 4 gland which has in-
Table 1. Baseline clinical characteristics of participants

\begin{tabular}{|c|c|c|c|}
\hline \multirow{2}{*}{ Variable/Case } & \multicolumn{2}{|c|}{ SBT } & \multirow{2}{*}{$\frac{\mathrm{CON}}{\mathrm{C}}$} \\
\hline & A & B & \\
\hline Age (yr) & 74 & 77 & 81 \\
\hline BMI $\left(\mathrm{kg} / \mathrm{m}^{2}\right)$ & 28.5 & 25.1 & 24.0 \\
\hline Education (yr) & $<12$ & $<12$ & $\geq 12$ \\
\hline IPAQ (MET-min/week) & 1173 & 2448 & 796 \\
\hline Surgery & Yes & No & No \\
\hline Comorbidities & DM & DM & HTN \\
\hline ADT duration (mo) & 50 & 12 & 15 \\
\hline Gleason score & 8 & 8 & 7 \\
\hline PSA (ng/m) & .85 & .16 & .07 \\
\hline Testosterone (ng/m) & $<.1$ & .22 & $<.1$ \\
\hline
\end{tabular}

SBT, strength and balance training; CON, control; yr, year; BMI, body mass index; DM, diabetes mellitus; HTN, hypertension; mo, month; min, minute; $B M D$, bone mineral density; IPAQ, international physical activity questionnaires; MET, metabolic equivalent of task; PSA, prostate-specific antigen.

vaded one or both of the seminal vesicles 8 years ago (T3c N0 M0) eight years prior to study. But he did not undergo any surgical operation, and started ADT with $3.6 \mathrm{mg}$ of Goserelin since June 2018. Thus, he had history of 12-month ADT prior to our study. Beside that DM was noted in his medical history. Although he was not a smoker but he stated he was a regular alcohol drinker. He reported us that he had habit of doing exercise regularly once a week and according to IPAQ his level of physical activity during one week before beginning our study was categorized as "moderate" which was 2,448 MET-minutes per week. When we asked him about motivation to participate in this study he also mentioned doctor was the one who recommended him.

Case $\mathrm{C}$ was an 81-year-old man, diagnosed with grade 3 gland in 2018. Following a transurethral resection of bladder tumor surgery, he began ADT with $3.6 \mathrm{mg}$ of Goserelin since March 2018 which had been increased to $10.8 \mathrm{mg}$ over a year. He also diagnosed with HTN as a comorbidity. He noted he is not a smoker but he drinks alcohol often. He stated he still does his carrier and do regular exercise habit more than once a week; but his level of physical activity on one week before starting the intervention categorized as "low" by IPAQ which was equal to 796 MET-minutes per week. He asserted he does exercise by his own so that was the reason he did not attend our exercise program.

\section{INTERVENTION}

All participants received physical activity diary and written educational materials about PCa and importance of exercise in cancer survivorship; then we conducted an 8-week intervention which SBT group 
participated in 16-session supervised exercise training with using resistance band (Thera-Band ${ }^{\circledR}$ ) while CON subject advised to follow the provided stretching training program at home. The SBT program (Fig. 1) consisted of 2 sets with 8-10 repetitions of balance and stability exercise with supports of barre (e.g., line walking, standing on one leg, leg raise, hip airplane, knee cross, single leg squat, heel raise, single leg balance and clock exercise), followed by 2 sets of strength training which mostly focused on major muscles of lower body (e.g., squat, lateral walk, good morning, leg press, bent knee plantar flexion, calf raise, kickback, calf press, seated row, straight leg raise, one leg circle, bridge). The average rating of perceived exertion was 13 (somewhat hard) which equivalents to moderate intensity [10]. We began exercise program with the green colored resistance band and modified intensity of the training by progression of every movements as well as upgrading the level of band to blue color.

All subjects were assessed for clinical and anthropometric assessments within one week before and after the intervention. We recorded data total body composition and BMD and T-score by whole body scan using dual energy x-ray absorptiometry (DXA, Hologic Discovery W, Waltham, MA, USA) machine. For evaluating physical functioning performance following testes were done; (a) the short physical performance battery (SPPB) test which is an objective assessment tool for lower extremity function combining balance, gait speed and chair stand; (b) the FMS ${ }^{\mathrm{TM}}$ test includes a total of seven fundamental movements that requires a balance of mobility and stability; (c) time to perform 6-m backward walk test which is designed to assess dynamic balance, and (d) TUG test which is aimed to evaluate functional balance. We used Cybex Norm ${ }^{\circledR}$ isokinetic dynamometer (Cybex International Inc., New York, NY, USA) machine to assess lower extremity strength through subject's preferred knee extension and flexion at angular velocity of $60 \%$ second. We also recorded data of self-reported falls efficacy scale-international (FES-I) for evaluating balance confidence, FACIT-F to assess fatigue, and FACT-P to evaluate patient's health related QOL. To examine safety of exercise we received blood variables before and after period of intervention. Results of each subject were expressed as difference between data of pre-test and post-test.

Ethical approval was granted from Institutional Review Board (IRB) of Seoul National University Boramae Medical Center (SNUIRB No. 262014-126) and written informed consent obtained from study participants.

\section{RESULTS}

Despite case C, BMD and its level which remarked as T-score improved in case A and B after the 8-week SBT program. However, muscle mass decreased in both Case A \& B; while it increased in case C. Also, slight increase of body fat had recorded in case B \& case C (Table 2).

Results of all physical functioning tests are presented in Fig. 2. All cases showed improvement in SPPB. The score of FMS, TUG, and Backwards walk test improved in case A \& B. However, no change was observed in FMS score of case $\mathrm{C}$ and his balance performance decreased over the period of intervention.
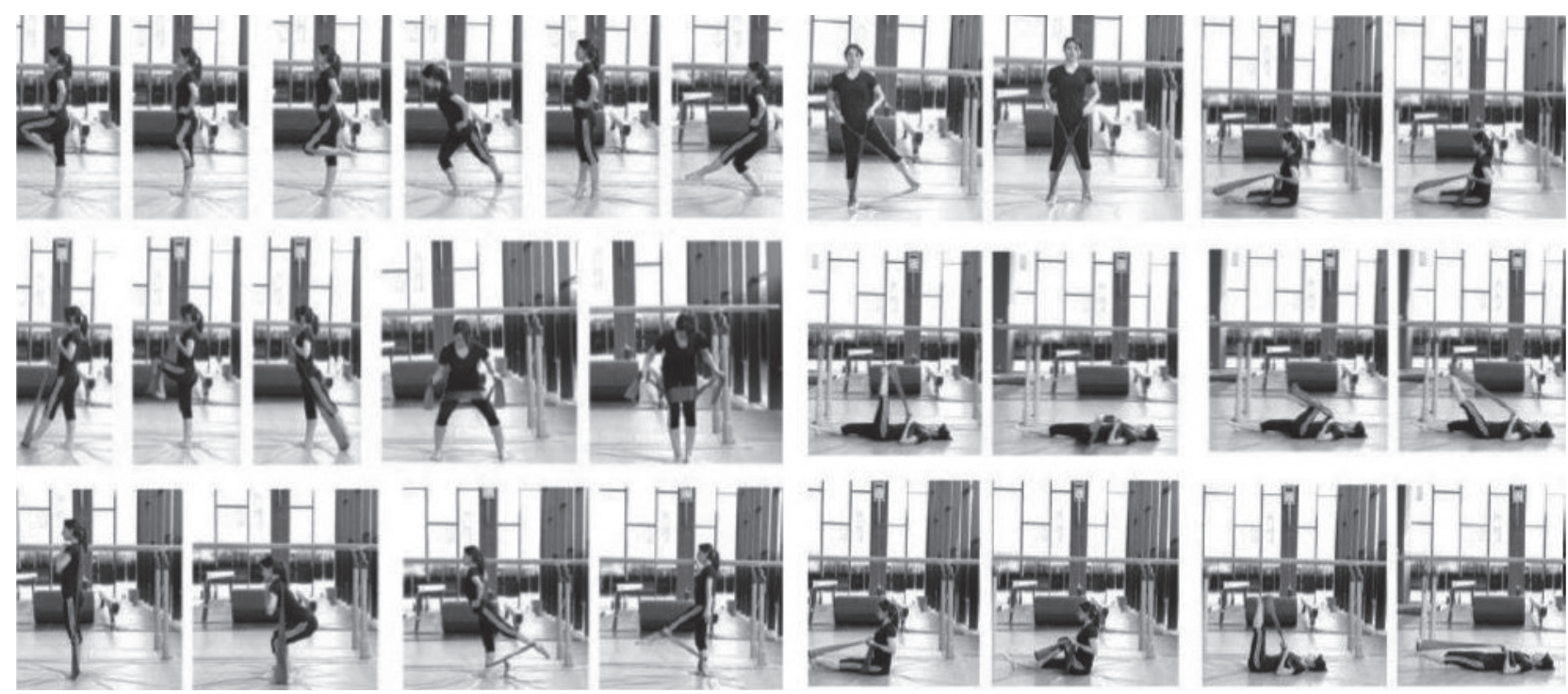

Fig. 1. Strength \& balance exercise program for prostate cancer patients receiving androgen deprivation therapy. 

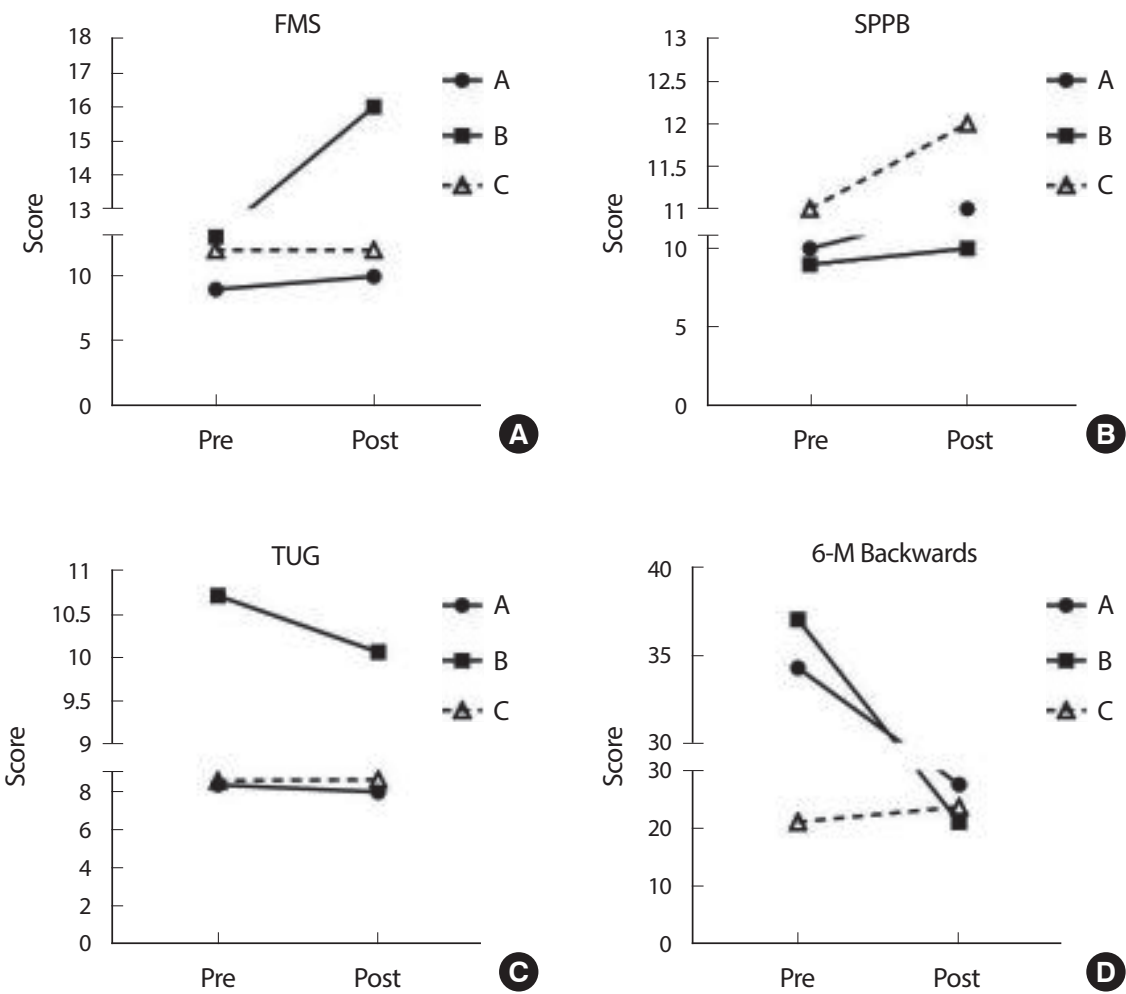

Fig. 2. The effects of 8-week strength and balance training on physical functioning performance of prostate cancer with Androgen deprivation therapy; (A) changes of functional limitation \& asymmetries (B) changes of lower extremity function; (C) changes of functional balance; (D) changes of dynamic balance. Strength and balance training group $(n=2): A \& B$; Control subject $(n=1): C$.

Table 2. Change of bone mineral density

\begin{tabular}{|c|c|c|c|c|}
\hline \multirow{2}{*}{ Variable } & \multirow{2}{*}{ Test } & \multicolumn{2}{|c|}{ SBT } & \multirow{2}{*}{$\frac{\mathrm{CON}}{\mathrm{C}}$} \\
\hline & & A & B & \\
\hline \multirow[t]{3}{*}{ Leg BMD $\left(\mathrm{g} / \mathrm{cm}^{2}\right)$} & Pre & 1.1775 & 1.214 & 1.2245 \\
\hline & Post & 1.1905 & 1.236 & 1.202 \\
\hline & Dif & .013 & .022 & -.225 \\
\hline \multirow[t]{3}{*}{ Total BMD $\left(\mathrm{g} / \mathrm{cm}^{2}\right)$} & Pre & 1.079 & 1.241 & 1.075 \\
\hline & Post & 1.081 & 1.251 & 1.07 \\
\hline & Dif & 0.002 & 0.01 & -.005 \\
\hline \multirow[t]{3}{*}{ T-score } & Pre & -1.2 & 0.4 & -1.3 \\
\hline & Post & -.3 & 0.5 & -1.3 \\
\hline & Dif & .9 & 0.1 & 0 \\
\hline \multirow[t]{3}{*}{ Muscle Mass (g) } & Pre & 50706.5 & 43147 & 37321.3 \\
\hline & Post & 50142.1 & 42891.2 & 37535.2 \\
\hline & Dif & -564.4 & -255.8 & 213.9 \\
\hline \multirow[t]{3}{*}{ Fat (\%) } & Pre & 38.35 & 28.95 & 33.4 \\
\hline & Post & 38.3 & 29.3 & 33.6 \\
\hline & Dif & -.05 & .35 & .2 \\
\hline
\end{tabular}

$\mathrm{SBT}$, strength and balance training; CON, control; BMD, bone mineral density; Dif, difference between pre-test \& post-test.

To assess the changes in lower muscle strength, we compared the normalized value of relative isokinetic muscle strength and power of each cases before and after the intervention (Fig. 3). The control case $\mathrm{C}$ had
Table 3. Changes of self-reported psychological variables

\begin{tabular}{llrrrr}
\hline \multirow{2}{*}{ Variable } & Test & \multicolumn{3}{c}{ SBT } & CON \\
\cline { 3 - 4 } \cline { 5 - 5 } & & A & B & C \\
\hline FACT-P & Pre & 20.6 & 21 & & 25.2 \\
& Post & 22 & 26.2 & & 27 \\
FACIT-F & Dif & 1.4 & 5.2 & & 1.8 \\
& Pre & 27 & 16 & & 48 \\
& Post & 44 & 19 & & 45 \\
FES-I & Dif & 17 & 3 & \\
& Pre & 22 & 19 & \\
& Post & 20 & 19 & 16 \\
& Dif & -2 & 0 & \\
\hline
\end{tabular}

SBT, strength and balance training; CON, control; FACT-P, functional assessment of cancer therapy-prostate; FACIT-F, functional assessment of chronic illness therapy-fatigue; FES-I, falls efficacy scale-international; Dif, difference between pre-test \& post-test.

dramatically decline in muscle strength and power of knee and only case B showed improvement in favor of SBT program.

According to the results of self-reported psychological questioners, overall score of FES-I questionnaire showed a modest improvement only in case A after 8-week intervention. Subjects of SBT group reported improvement in the FACIT-F questionnaire, while control subject ex- 
Muscle power

Knee extensor (Average power per repetition/\% BW)

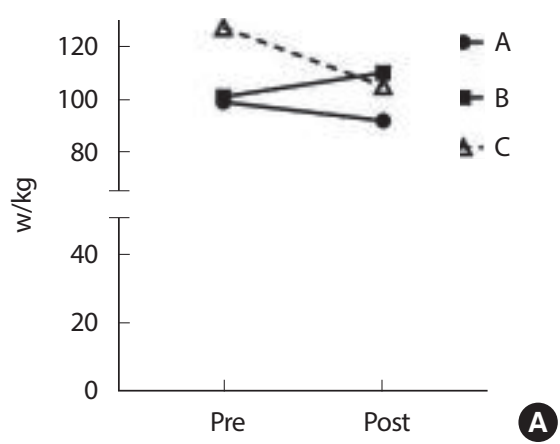

Muscle strength Knee extensor (Peak torque/\% BW)

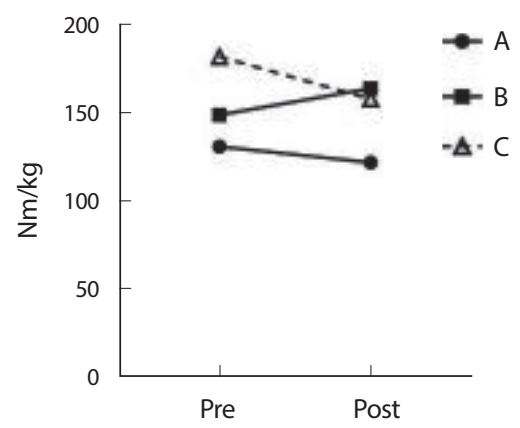

Muscle power

Knee flexor (Average power per repetition/\% BW)

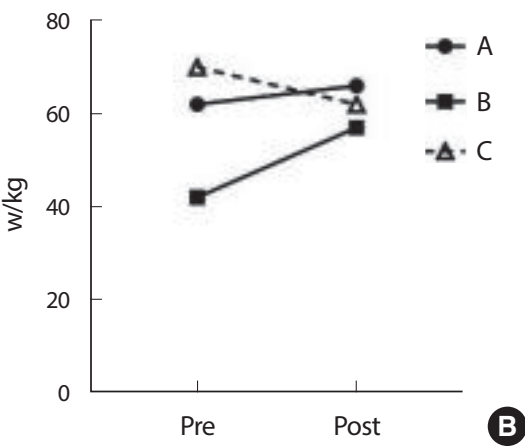

Muscle strength Knee flexor (Peak torque/\% BW)

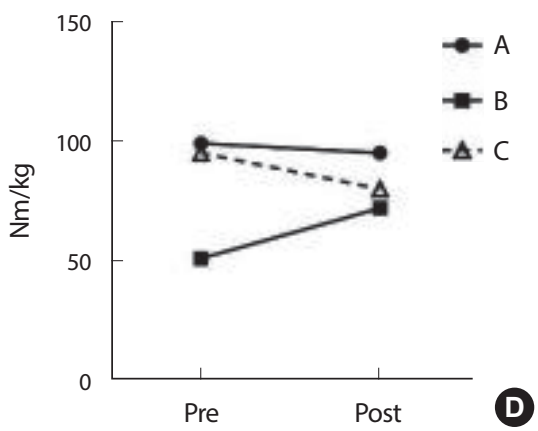

Fig. 3. The effects of 8-week strength and balance training on lower muscle strength and power of Prostate cancer men with androgen deprivation therapy; (A\&B) changes of lower muscle power; (C\&D) changes of lower muscle strength; Strength and balance training group $(n=2)$ : A\&B; Control subject $(n=1): C$.

pressed more fatigue symptoms after the 8 -week intervention. And all participants of this study had improvement in their health related QOL (Table 3).

\section{DISCUSSION}

Recently increased risk of falls in PCa patients fallowing ADT has been taken into consideration [2], yet there is no tailored fall-prevention exercise reported for this population. The evidence implies that ADT decreased BMD and it is detrimental to bone health [4]. But little is known about proper exercise program which preserve bone health of ADT patients, however increased BMD following a 12-week exercise intervention with resistance band have been observed in sarcopenic elderly women [11] which also supports our exercise protocol to have the potential to turn over negative effects of ADT on bone mineral density in PCa patients (Table 2).

Except examining bone health, clinical assessment of fall risk factors such as functional performance and gait are often quantified in older population [12]. In this study we observed a slight improvement in functional limitation through FMS test of exercise participants (Fig. 2A), while the score of SPPB which is wildly used for evaluating lower extremity function in older adults did not differ (Fig. 2B). We assume that might be due to physically active lifestyle of our subjects which were greater than physical activity recommendation for older adults, therefore their respond to SPPB test before starting the intervention was quite higher that older age population [10].

Regarding to dynamic balance domain of functioning performance we asked subjects to perform TUG and 6-m backwards walk which are commonly-used tasks in older adults and cancer population. In addition we used data of patients' self-reported FES-I questionnaire to assess fear of fall and balance confidence [13]. Similar to results of our study which indicate improvement of balance (Fig. 2C, D), De Bruin, E. D. and K. Murer study also demonstrated the effectiveness of 12 weeks of SBT to improve dynamic posture balance on older adults [14]. Notably, fear of 
falling has been shown to be associated with negative consequences such as avoidance of activities of daily living, falling, depression, and lower QOL [15] which also improved in SBT group (Table 3).

In this study we could not find similar tendency in subjects of SBT group in variables of muscle mass, strength and power after the 8-week exercise program which was relatively a short period of intervention (Fig. 3). It seems at least 12 weeks of strength training or combination of aerobic and strength training is required to impact muscle strength in prostate cancer patients receiving hormone therapy [16]. Although the possible relation of muscle strength and balance performance in elderly have been documented, yet much controversy. Several studies indicated no interaction of strength and balance by reporting less or no difference in strength between fallers and non-fallers [17] and then explained the enhancement of functional and balance performance regardless of muscle strength by possible improvement in sensory system (e.g., neuromuscular, visual, somatosensory and vestibular) which gradually decreases with aging and therefore could affect statistic and dynamic performance [18]. Thus, we assume that the reason why participants in our study showed improvement in performance resulted from the characteristics of the exercise intervention which contained postural control and stability exercise with less support and challenging the alignment of the body's center of gravity with regard to base of support.

The results of our study on two participants showed the 8-weeks SBT intervention had positive effect on some of falls risk factors such as bone health, functional and balance performance, as well as QOL using resistance band which is a comfortable and convenient equipment of training for elderly. We also presented data of one control case but we did not limit other physical activities of all participants which may had contribution in our outcome. Due to small number of participants we could not apply further quantitative analysis methods to make any conclusion on the efficacy of the intervention. We could not declare the effects of SBT on lower body strength and power. Perhaps the period of our intervention was not long enough to impact muscle components. Further studies with large sample size and longer period of intervention should apply this type of multicomponent training to discuss the effect on muscle health and fall risk factors. A follow-up study after the period of exercise also is necessary to conclude the effects of intervention on reducing rate of falls which is another limitation of our study that we could not apply. Optimal time and duration of exercise training to prevent falls in PCa is also another issue that still is not confirmed. In fact, several studies reported that exercise can improve individual frailty components, but we do not yet know what type of exercise can be more beneficial to reduce overall falls status in PCa on ADT.

\section{CONFLICT OF INTEREST}

No potential conflict of interest relevant to this article was reported. Availability of data and materials.

The datasets analyzed during the current study are available if requested.

\section{AUTHOR CONTRIBUTION}

Conceptualization: P Jamrasi, K Lee, K Noh; Data curation: P Jamrasi, J Park, H Jeong, S Yoo; Formal analysis: P Jamrasi; Methodology: P Jamrasi, J Park, H Jeong, S Yoo; Project administration: P Jamrasi; Visualization: P Jamrasi; Writing-original draft: P Jamrasi; Writing-review \& editing: P Jamrasi, K Lee, K Noh, J Park, H Jeong, S Yoo.

\section{ORCID}

WookSong https://orcid.org/0000-0002-8825-6259

Parivash Jamrasi https://orcid.org/0000-0002-5262-8655

Kyunghee Lee https://orcid.org/0000-0002-5612-6353

Kyoungmin Noh https://orcid.org/0000-0002-0146-5868

Juhyun Park https://orcid.org/0000-0002-5549-9756

Hyeon Jeong https://orcid.org/0000-0002-0744-1402

Sangjun Yoo https://orcid.org/0000-0003-4992-7053

\section{REFERENCES}

1. Bray F, Ferlay J, Soerjomataram I, Siegel RL, Torre LA, et al. Global cancer statistics 2018: GLOBOCAN estimates of incidence and mortality worldwide for 36 cancers in 185 countries. CA Cancer J Clin. 2018;68(6):394-424.

2. Winters-Stone KM, Moe E, Graff JN, Dieckmann NF, Stoyles S, et al. Falls and frailty in prostate cancer survivors: current, past, and never users of androgen deprivation therapy. J Am Geriatr Soc. 2017;65(7): 1414-9.

3. Wu FJ, Sheu SY, Lin HC, Chung SD. Increased fall risk in patients receiving androgen deprivation therapy for prostate cancer. urology. 2016;95:145-50. 
4. Kim DK, Lee JY, Kim KJ, Hong N, Kim JW, et al. Effect of androgendeprivation therapy on bone mineral density in patients with prostate cancer: a systematic review and meta-analysis. J Clin Med. 2019;8(1).

5. Shahinian VB, Kuo YF, Freeman JL, Goodwin JS. Risk of fracture after androgen deprivation for prostate cancer. N Engl J Med. 2005;352(2): $154-64$.

6. Cadore EL, Rodríguez-Mañas L, Sinclair A, Izquierdo M. Effects of different exercise interventions on risk of falls, gait ability, and balance in physically frail older adults: a systematic review. Rejuvenation Res. 2013;16(2):105-14

7. Piercy KL, Troiano RP, Ballard RM, Carlson SA, Fulton JE, et al. The physical activity guidelines for americans. JAMA. 2018;320(19):202028.

8. Orr R, Raymond J, Singh MF. Efficacy of progressive resistance training on balance performance in older adults. Sports Med. 2008;38(4): 317-43.

9. De Bruin ED, Murer K. Effect of additional functional exercises on balance in elderly people. Clin Rehabil. 2007;21(2):112-21.

10. Riebe D, Ehrman JK, Liguori G, Magal M, ACSM's guidelines for exercise testing and prescription. American College of Sports Medicine; 2018.

11. Huang SW, Ku JW, Lin LF, Liao CD, Chou LC, et al. Body composition influenced by progressive elastic band resistance exercise of sarcopenic obesity elderly women: a pilot randomized controlled trial. Eur J Phys Rehabil Med. 2017;53(4):556-63.
12. Mion LC, Chandler AM, Waters TM, Dietrich MS, Kessler LA, et al. Is it possible to identify risks for injurious falls in hospitalized patients? Jt Comm J Qual Patient Saf. 2012;38(9):408-AP403.

13. Williams AD, Bird ML, Hardcastle SGK, Kirschbaum M, Ogden KJ, et al. Exercise for reducing falls in people living with and beyond cancer. Cochrane Database Syst Rev. 2018. doi:10.1002/14651858.CD011687. pub2(10).

14. Gusi N, Carmelo Adsuar J, Corzo H, Del Pozo-Cruz B, Olivares PR, et al. Balance training reduces fear of falling and improves dynamic balance and isometric strength in institutionalised older people: a randomised trial. J Physiother. 2012;58(2):97-104.

15. Rajagopalan R, Litvan I, Jung TP. Fall prediction and prevention systems: recent trends, challenges, and future research directions. sensors (Basel, Switzerland). 2017;17(11):2509.

16. Cormie P, Galvao DA, Spry N, Joseph D, Chee R, et al. Can supervised exercise prevent treatment toxicity in patients with prostate cancer initiating androgen-deprivation therapy: a randomised controlled trial. BJU Int. 2015;115(2):256-66.

17. Oddsson LIE, Boissy P, Melzer I. How to improve gait and balance function in elderly individuals-compliance with principles of training. Eur Rev Aging Phys Act. 2007;4(1):15-23.

18. Lesinski M, Hortobágyi T, Muehlbauer T, Gollhofer A, Granacher U. Effects of balance training on balance performance in healthy older adults: a systematic review and meta-analysis. Sports Med. 2015;45 (12):1721-38. 